\title{
New nomenclatural combinations in Pseudopodospermum (Asteraceae)
}

\author{
V. M. Vasjukov*, S. V. Saksonov \\ Institute of Ecology of the Volga river basin RAS - Branch of the Samara Federal Research Center RAS, Komzina str., 10, \\ Togliatti,445003,Russian Federation.E-mail: vvasjukov@yandex.ru \\ * Corresponding author
}

Keywords: new combinations, nomenclature, Pseudopodospermum, Scorzonera.

Summary. New combinations are validated: Pseudopodospermum glastifolium (Willd.) Vasjukov et Saksonov, comb. nov., Pseudopodospermum tauricum (M. Bieb.) Vasjukov et Saksonov, comb. nov. (Asteraceae).

\section{Новые номенклатурные комбинации в роде Pseudopodospermum (Asteraceae)}

\author{
В. М. Васюков, С. В. Саксонов \\ Институт экологии Волжского бассейна РАН - филиал Самарского федерального исследовательского иентра РАН, \\ ул. Комзина, 10, г. Тольятти, 445003, Россия
}

Ключевые слова: новые комбинации, номенклатура, Pseudopodospermum, Scorzonera.

Аннотация. Обнародованы новые номенклатурные комбинации: Pseudopodospermum glastifolium (Willd.) Vasjukov et Saksonov, comb. nov., Pseudopodospermum tauricum (M. Bieb.) Vasjukov et Saksonov, comb. nov. (Asteraceae).

The genus Scorzonera L. s. 1. (Asteraceae, Cichorieae) comprises 180-190 species and its circumscription has long been the subject of debate. Available molecular phylogenetic analyses affirmed the polyphyly in its wide sense. M. A. Zaika et al. (2020) provided a re-evaluation of Scorzonera and other related genera based on carpological (including anatomical) and extended molecular phylogenetic analyses.

Below we present two new combinations in one of Scorzonera segregates, Pseudopodospermum (Lipsch. et Krasch.) Kuth.
Pseudopodospermum glastifolium (Willd.) Vasjukov et Saksonov, comb. nov. $\equiv$ Scorzonera glastifolia Willd. 1803, Sp. Pl., ed. 4 [Willdenow] 3(3): 1499.

Pseudopodospermum tauricum (M. Bieb.) Vasjukov et Saksonov, comb. nov. $\equiv$ Scorzonera taurica M. Bieb. 1808, Fl. Taur.-Caucas. 2: 234.

\section{Acknowledgements}

The authors are grateful for valuable advice to A. P. Sukhorukov.

\section{REFERENCES}

Zaika M. A., Kilian N., Jones K., Krinitsina A. A., Nilova M. V., Speranskaya A. S., Sukhorukov A. P. 2020. Scorzonera sensu lato (Asteraceae, Cichorieae) - taxonomic reassessment in the light of new molecular phylogenetic and carpological analyses. PhytoKeys 137: 1-85. DOI: $10.3897 /$ phytokeys. 137.46544 